\title{
Aspectos farmacológicos do idoso: uma revisão integrativa de literatura
}

\author{
Pharmacological aspects of elderly: an integrative literature review
}

\author{
Henrique Souza Barros de Oliveira ${ }^{1}$, Maria Luiza Galoro Corradi ${ }^{2}$
}

Oliveira HSB, Corradi MLG. Aspectos farmacológicos do idoso: uma revisão integrativa de literatura / Pharmacological aspects of elderly: an integrative literature review. Rev Med (São Paulo). 2018 mar.-abr.;97(2):165-76.

RESUMO: Os idosos constituem o grupo populacional que mais cresce de forma acelerada e descontrolada no Brasil, representando $14,3 \%$ da população geral. Associado a isto e ao aumento da expectativa de vida, mudança do padrão de adoecimento do pais e a medicalização no idoso, temas como a farmacologia do indivíduo idoso tornam-se essenciais na formação de um profissional da área da saúde. O processo normal do envelhecimento possui particularidades capazes de modificar as etapas da farmacocinética e a farmacodinâmica de um medicamento, predispondo o idoso às Reações Adversas a Medicamentos (RAM), cascata iatrogênica, uso de Medicamentos Potencialmente Inapropriados (MPI) e polifarmácia, processo do adoecimento, quedas e fraturas, delirium, hospitalização, institucionalização e até à morte. Baseado nisso, instrumentos ou listas de medicamentos considerados inadequados para uso em idosos foram criadas, cada uma com particularidades da comercialização de seu país. Visto isso, entende-se que o assunto é de extrema relevância na pratica clínica, e, quando utilizado de forma interdisciplinar, melhora a qualidade de serviços, formação de profissionais da área da saúde e permite a prevenção de eventos iatrogênicos.

Descritores: Idoso; Farmacologia. Envelhecimento; Lista de medicamentos potencialmente inapropriados/utilização; Efeitos colataterais e reações adversas à medicamentos; Serviços de saúde para idosos.

\begin{abstract}
The elderly are the fastest growing population group in Brazil, accounting for $14.3 \%$ of the population. Associated with this and with the increase in life expectancy, changes in the country's pattern of illness and the medicalization of aging, issues such as pharmacology in elderly have become essential topics in the health professionals' training. The normal aging process has particularities that can modify the pharmacokinetic and pharmacodynamic properties of drugs, predisposing elderly to Adverse Drug Reactions (ADRs), iatrogenesis, use of Potentially Inappropriate Medications (PIM), polypharmacy, illness processes, falls and fractures, delirium, hospitalization, institutionalization and even death. Based on this, instruments or lists of medications considered inappropriate for elderly were created, each with particularities of the medication market of each country. Therefore, this subject is extremely relevant in clinical practice and when it is used in an interdisciplinary way, it improves the quality of services and the training of health professionals and allows the prevention of iatrogenic events.
\end{abstract}

Keywords: Aged; Pharmacology; Aging; Potentially inapropriate medications/utilization; Drug-related side effects and adverse reactions; Health services for the aged.

1. Centro Universitário São Camilo (CUSC-SP), São Paulo, SP, BR. Discente do curso de Medicina, interno no Hospital Geral de Carapicuíba (HGC), Diretor da Liga de Estudos do Processo do Envelhecimento (LEPE) e Pesquisador vigente do CNPq/PIBIC no Instituto Sírio-Libanês de Ensino e Pesquisa (IEP-HSL). E-mail: heeenry.barros@icloud.com; ORCID: https://orcid.org./00000002-9067-8581.

2. Faculdade de Medicina do Centro Universitário São Camilo (CUSC-SP), São Paulo, SP, BR. Médica, Geriatra pela Santa Casa de Misericórdia de São Paulo (SCM/SP), titulada pela Sociedade Brasileira de Geriatria e Gerontologia (SBGG) e Mestranda em Educação nas Profissões da Saúde pela Pontifícia Universidade Católica de São Paulo (PUC/SP). Docente da Faculdade do CUSCSP, Coordenadora da enfermaria e da Residência Médica de Clínica Médica do Hospital Geral de Carapicuíba (HGC). E-mail: malugaloro@gmail.com.

Endereço para correspondência: Henrique Souza Barros de Oliveira. Rua José Martins Borges, 249 - Jd. Leonor Mendes de Barros. São Paulo, SP, Brasil. E-mail: heeenry.barros@icloud.com. 


\section{INTRODUÇÃO}

Arganização Mundial de Saúde (OMS) define saúde como "Um estado de completo bem-estar físico, mental e social e não somente ausência de afecções e enfermidades". Entretanto, a maioria da população idosa possui uma ou mais Doenças Crônicas Não Transmissíveis (DCNT), que muitas vezes não estão associadas a limitações funcionais, permitindo assim que desempenhem o seu papel social normalmente. Baseado nisso, a Política Nacional de Saúde da Pessoa Idosa (PNSPI) conceituou saúde do idoso pela "condição de autonomia e independência do que pela presença ou ausência de doenças orgânica".

$\mathrm{O}$ envelhecer corresponde a um processo sequencial, individual, acumulativo, irreversível, universal, não patológico, próprio a todos os membros de uma espécie, onde há uma deterioração de um organismo maduro. A partir desse processo, o organismo torna-se, cada vez mais, incapaz de responder de forma rápida e abrupta aos estresses do meio ambiente, aumentando a sua possibilidade de morte ${ }^{2}$.

Sabe-se que o processo do envelhecimento associado às doenças crônico-degenerativas é fator para maior demanda aos serviços de saúde, o que corrobora para o uso de polifarmácia, Riscos Adversos aos Medicamentos (RAM) e aos outros eventos iatrogênicos ${ }^{3-6}$. Atualmente, os idosos constituem o grupo populacional que mais crescem no Brasil e são os maiores consumidores de medicamentos do mundo. Graças a isso, o país se tornou o quarto mercado mundial com maior consumo de medicamentos, sendo o setor farmacêutico brasileiro um dos maiores do mundo ${ }^{7}$.

Em consequência do uso prescrito e não prescrito (automedicação), as reações adversas aos medicamentos tornaram-se uma das principais causas de admissão em serviços de saúde e um grande problema de saúde pública. A partir disto, o presente estudo destaca a importância de estudar o impacto do processo do envelhecimento e sua influência sobre a farmacologia no organismo do indivíduo idoso.

\section{METODOS}

Este é um estudo exploratório do tipo revisão de literatura, que tem como objetivo abordar o impacto da senescência sobre a farmacologia do idoso. A busca de estudos realizou-se nas bases de dados NCBI/PubMed (National Center for Biotechnology Information), SciELO (Scientific Eletronic Lirary Online) e Lilacs - Bireme (Literatura Latino-Americana e do Caribe em Ciências da Saúde) com os seguintes descritores: "Farmacology", "Polypharmacy", "Potentially Inappropriate Medications", "Drug-related Adverse Reactions", "Placebo and nocebo effects", "elderly" e "aging" e seus respectivos correspondentes em português/espanhol.

O presente estudo limitou-se apenas ao tipo de acesso às publicações, sendo selecionados os estudos considerados de livre acesso nas bases de dados citadas anteriormente. A partir disso, somente os artigos com resumo/abstract que contemplasse o assunto a ser estudado foram utilizados nesta pesquisa.

\section{RESULTADOS}

Ao final, foram incluídos 18 estudos para integrar este artigo de revisão. A Tabela 1 apresenta a distribuição por autoria, país de origem, ano de publicação, título, revista e base de dados dos estudos selecionados.

Tabela 1 - Distribuição dos estudos selecionados segundo autoria, país de origem, ano de publicação, título, revista e a base de dados

\begin{tabular}{|c|c|c|c|c|c|}
\hline \multirow{2}{*}{$\begin{array}{l}\text { Autor/ano, } \\
\text { País de origem }\end{array}$} & \multirow{2}{*}{ Titulo } & \multirow{2}{*}{ Revista } & \multicolumn{3}{|c|}{ Base de dados } \\
\hline & & & Pubmed & SciELO & Lilacs \\
\hline $\begin{array}{l}\text { Silva et al., } 2011 \\
\text { Brasil }\end{array}$ & $\begin{array}{l}\text { Metabolismo mitocondrial, radicais livres e } \\
\text { envelhecimento }\end{array}$ & Rev Bras Geriatr Gerontol & - & 01 & - \\
\hline $\begin{array}{l}\text { Silva et al., } 2012 \\
\text { Brasil }\end{array}$ & Polifarmácia em geriatria & Rev AMRIGS & - & - & 01 \\
\hline $\begin{array}{l}\text { Manso et al., } 2015 \\
\text { Brasil }\end{array}$ & $\begin{array}{l}\text { Prescrição inadequada de medicamentos a } \\
\text { idosos portadores de doenças crônicas em } \\
\text { um plano de saúde no município de São } \\
\text { Paulo, Brasil }\end{array}$ & Rev Bras Geriatr Gerontol & - & 01 & - \\
\hline $\begin{array}{l}\text { Carvalho, } 2007 \\
\text { Brasil }\end{array}$ & $\begin{array}{l}\text { A polifarmácia em idosos no município de } \\
\text { São Paulo - Estudo SABE - Saúde, Bem- } \\
\text { estar e Envelhecimento }\end{array}$ & - & - & - & 01 \\
\hline $\begin{array}{l}\text { Secoli, } 2010 \\
\text { Brasil }\end{array}$ & $\begin{array}{l}\text { Polifarmácia: interações e reações adversas } \\
\text { no uso de medicamentos por idosos }\end{array}$ & Rev Bras de Enferm & - & 01 & - \\
\hline $\begin{array}{l}\text { Sousa-Munoz et al., } 2012 \\
\text { Brasil }\end{array}$ & $\begin{array}{l}\text { Prescrições geriátricas inapropriados e } \\
\text { polifarmacoterapia em enfermarias de } \\
\text { clínica médica de um hospital-escola }\end{array}$ & Rev Bras Geriatr Gerontol & - & 01 & - \\
\hline $\begin{array}{l}\text { Quinalha et al., } 2010 \\
\text { Brasil }\end{array}$ & $\begin{array}{l}\text { Instrumentos para avaliação da } \\
\text { farmacoterapia do idoso: uma revisão }\end{array}$ & Rev Bras Geriatr Gerontol & - & 01 & - \\
\hline
\end{tabular}


Tabela 1 - Distribuição dos estudos selecionados segundo autoria, país de origem, ano de publicação, título, revista e a base de dados Tontinuação

\begin{tabular}{|c|c|c|c|c|c|}
\hline \multirow{2}{*}{$\begin{array}{l}\text { Autor/ano, } \\
\text { País de origem }\end{array}$} & \multirow{2}{*}{ Título } & \multirow{2}{*}{ Revista } & \multicolumn{3}{|c|}{ Base de dados } \\
\hline & & & Pubmed & SciELO & Lilacs \\
\hline $\begin{array}{l}\text { American Geriatrics } \\
\text { Society, } 2012 \\
\text { EUA }\end{array}$ & $\begin{array}{l}\text { American Geriatrics Society updated } \\
\text { Beers criteria for potentially inappropriate } \\
\text { medication use in older adults }\end{array}$ & J Am Geriatr Soc & 01 & - & - \\
\hline $\begin{array}{l}\text { American Geriatrics } \\
\text { Society, } 2015 \\
\text { EUA }\end{array}$ & $\begin{array}{l}\text { American Geriatrics Society } 2015 \text { updated } \\
\text { Beers criteria for potentially inappropriate } \\
\text { medication use in older adults }\end{array}$ & J Am Geriatr Soc & 01 & - & - \\
\hline $\begin{array}{l}\text { Silveira et al., } 2009 \\
\text { Espanha }\end{array}$ & $\begin{array}{l}\text { Prescripción inapropriada de medicamentos } \\
\text { em los pacientes mayores: los critérios } \\
\text { STOPP/START }\end{array}$ & Rev Esp Geriatr Gerontol & 01 & - & - \\
\hline $\begin{array}{l}\text { O’Mahony et al., } 2014 \\
\text { Inglaterra }\end{array}$ & $\begin{array}{l}\text { STOPP/START criteria for potentially } \\
\text { inappropriate prescribing in older people: } \\
\text { version } 2\end{array}$ & Age and Ageing & 01 & - & - \\
\hline $\begin{array}{l}\text { Holt et al., } 2010 \\
\text { Alemanha }\end{array}$ & $\begin{array}{l}\text { Potentially inappropriate medications in the } \\
\text { elderly: the PRISCUS list }\end{array}$ & Deutsches Arzteblatt Int & 01 & - & - \\
\hline $\begin{array}{l}\text { Oliveira et al., } 2017 \\
\text { Brasil }\end{array}$ & $\begin{array}{l}\text { Consenso brasileiro de medicamentos } \\
\text { potencialmente inapropriados para idosos }\end{array}$ & Geriatr Gerontol Aging & - & 01 & - \\
\hline $\begin{array}{l}\text { Lopes et al., } 2016 \\
\text { Brasil }\end{array}$ & $\begin{array}{l}\text { Utilização de medicamentos potencialmente } \\
\text { inapropriados por idosos em domicilio }\end{array}$ & Cien Saúde Coletiva & - & 01 & - \\
\hline $\begin{array}{l}\text { Foster et al., } 2017 \\
\text { EUA }\end{array}$ & $\begin{array}{l}\text { Comparative "nocebo effects" in older } \\
\text { patients enrolled in câncer therapeutic trials: } \\
\text { Observations from a 446-patient cohort }\end{array}$ & Cancer & 01 & - & - \\
\hline $\begin{array}{l}\text { Cruz et al., } 2010 \\
\text { EUA }\end{array}$ & $\begin{array}{l}\text { Placebo and nocebo effects in randomized } \\
\text { double blind clinical trials of agentes for } \\
\text { the treatment of fatigue in advanced cancer } \\
\text { patients }\end{array}$ & Cancer & 01 & - & - \\
\hline $\begin{array}{l}\text { Hunter et al., } 2005 \\
\text { EUA }\end{array}$ & $\begin{array}{l}\text { Neurophysiologic correlates of side } \\
\text { effects in normal subjects randomized to } \\
\text { venlafaxine or placebo }\end{array}$ & Neuropsychopharmacology & 01 & - & - \\
\hline Total: & & & 09 & 07 & 02 \\
\hline
\end{tabular}

\section{DISCUSSÃO}

Um indivíduo por volta dos 30 a 40 anos atinge o máximo das suas funções orgânicas, sendo que entre os 40 a 50 há uma estabilização seguida de um declínio progresso a partir dos 60 anos de idade. Tais alterações funcionais, consideradas não patológicas e associadas a perda progressiva da reserva orgânica no organismo do idoso denomina-se senescência ou senectude. Trata-se de modificações fisiológicas que ocorrem com à passagem do tempo (p.ex.: surgimento de rugas ou cabelos brancos). Diferentemente da senilidade, definida como um processo patológico do envelhecimento, onde há um desequilíbrio homeostático do organismo, que eleva o risco de morbimortalidade no idoso ${ }^{3,4,6}$.

\section{Envelhecimento celular}

As alterações bioquímicas, moleculares e estruturais que ocorrem no organismo do idoso são denominadas de envelhecimento celular. Segundo a teórica dos radicais livres, o meio intracelular do organismo do idoso é diferenciado do jovem devido ao acúmulo de proteínas, lipídeos, carboidratos e DNA oxidados. Justificando assim, algumas alterações estruturais onde o transporte de íons e nutrientes é modificado na membrana plasmática, as mitocôndrias formam substancias indesejáveis durante o metabolismo celular decorrente da formação e acúmulo dessas substancias e, os ácidos ribonucleicos, localizados no núcleo celular, tornam-se mais condensados dificultando o mecanismo de reparo e divisão celular ${ }^{8}$.

Há ainda fatores como: (1) hormese induzida pela prática regular da atividade física, (2) restrição calórica, (3) ingesta de antioxidantes nutricionais e o (4) aumento da produção de antioxidantes celulares que promovem uma integridade e funcionalidade mitocondrial capaz de reduzir o estresse oxidativo e nitrosativo, favorecendo à redução do envelhecimento e do aumento da longevidade 8 .

No idoso, há ainda uma redução da quantidade 
total de água corpórea e dos componentes intracelulares e extracelulares, tornando-o mais susceptível aos graves distúrbios de perda de líquido e maior dificuldade para reposição volêmica perdida. Decorrente a isso, alterase a biodisponibilidade de drogas hidrossolúveis, tendo diminuição do seu volume de distribuição. Diferentemente das drogas lipossolúveis, que tem maior volume de distribuição, devido ao aumento na quantidade de tecido adiposo com o envelhecimento ${ }^{3,4,6}$.

Todos esses processos corroboram para a apoptose ou modificação da função celular, tornando os idosos mais vulneráveis ao processo de adoecimento ${ }^{3,4,6}$.

\section{Farmacocinética}

A absorção, distribuição, metabolismo e a excreção correspondem aos processos farmacocinéticos que sofrem modificações com o envelhecimento. Essas modificações geram alterações na concentração de alguns receptores. $\mathrm{O}$ fígado e os rins são os principais órgãos afetados na farmacologia do idoso, entretanto outras estruturas também sofrem modificações ${ }^{3,4,6,9}$.

Os idosos podem apresentar problemas desde a ingesta até a excreção, seja pela ausência de dentição e/ ou pela diminuição da produção salivar. Alguns fármacos podem ainda causar lesão gástrica (gastrite) por aderirem a mucosa local ou terem a absorção ineficiente devido a diminuição da secreção de ácido clorídrico, peristaltismo esofágico e gástrico, da perfusão do trato digestivo, a superfície absortiva e pela atividade de transporte ativo de membrana já discutido. Cabe ressaltar que, a diminuição do peristaltismo digestivo do idoso, favorece ao aumento do período de contato entre o fármaco e a superfície mucosa de absorção $0^{3,4,6,9}$.

Há uma diminuição sérica de proteínas plasmáticas no processo de envelhecer sendo a albumina uma destas, que tem como principal repercussão a maior fração livre de fármacos. Essa alteração sérica gera problemas de transporte de substâncias, favorecendo ao risco de intoxicação medicamentosa ${ }^{3,4,6,9}$.

No fígado ocorre uma redução significativa no número de hepatócitos e de massa hepática gerando uma diminuição do fluxo sanguíneo. Isso justifica o declínio em $30 \%$ da depuração hepática, diminuição do metabolismo de oxidação e conjugação, aumento na concentração plasmática de alguns fármacos (propranolol, verapamil, lidocaína). A imipramina, amtriptilina, morfina e meperidina são fármacos que não sofrem alterações plasmáticas decorrentes da função hepática ${ }^{3,4,6,9}$.

Baseado na diminuição do sistema enzimático do fígado, há uma redução na produção de metabolitos responsáveis por diminuírem a quantidade total dos fármacos em circulação e assim de interagirem com outros órgãos ${ }^{3,4,69}$.

Alguns fármacos podem ser metabolizados e excretados via hepato-intestinal, enquanto que outros sofrem excreção somente por via renal. Como consequência da senescência renal, ocorre perda de 30 a $40 \%$ de massa renal, diminuição dos $60 \%$ dos glomérulos, queda do fluxo sanguíneo, envelhecimento da cápsula glomerular, espessamento das paredes dos túbulos renais (atrofia tubular renal), diminuição do clearence de creatinina ( $1 \%$ ao ano), queda de 30 a 40\% da Taxa de Filtração Glomerular (TFG) e da secreção tubular. Decorrente a isso, a capacidade de eliminar os fármacos fica prejudicada, levando ao efeito prolongado dos fármacos de excreção renal. Inúmeros autores recomendam o uso da formula de Cockcroft e Gault antes de prescrever um fármaco com excreção renal ao idoso, principalmente antibióticos. Os idosos podem ainda precisar de reajuste de dose com o passar do tempo devido a deterioração funcional dos rins ${ }^{3,4,6,9}$.

Cabe ressaltar que, a redução da TFG é considerada a principal alteração farmacocinética do processo normal do envelhecimento de um indivíduo ${ }^{3,6}$.

A Tabela 2 mostra as principais alterações farmacocinéticas decorrente da senescência.

\section{Farmacodinâmica}

O declínio dos mecanismos homeostáticos e de algumas funções orgânicas do idoso estão intrinsecamente associadas às alterações de sensibilidade a diversos fármacos. A redução do fluxo sanguíneo cerebral, hipotensão ortostática, disfunções renal e intestinal, alteração da responsividade do reflexo barorreceptor associada ao envelhecimento, dificuldade de termorregulação, queda da capacidade cognitiva, alterações metabólicas e resposta imunitária diminuída são algumas destas funções orgânicas associadas $^{4,9}$.

Há ainda modificações na interação entre os fármacos e seus receptores, com consequente efeito final à ação do medicamento. Os receptores alfa e beta adrenérgicos, muscarínicos da acetilcolina e receptores GABA são alguns receptores associados aos diferentes efeitos final apresentados por idosos à medicamentos ${ }^{9}$.

Devido a esses e outros aspectos do processo do envelhecimento, a saúde do idoso deve ser abordada por uma avaliação integral e multidimensional, necessitando raciocínio clinico para as diferentes manifestações clinicas que um idoso pode apresentar por consequência de um fármaco. 
Tabela 2 - Alterações farmacocinéticas decorrente do processo normal do envelhecimento e as suas repercussões clínicas

\begin{tabular}{|c|c|c|}
\hline $\begin{array}{l}\text { Processos } \\
\text { farmacológicos }\end{array}$ & $\begin{array}{l}\text { Alterações no } \\
\text { envelhecimento }\end{array}$ & Repercussões clínicas \\
\hline \multirow{4}{*}{ Absorção } & $\begin{array}{l}\downarrow \text { Número de células de } \\
\text { absorção }\end{array}$ & Atrofia intestinal \\
\hline & $\uparrow \mathrm{pH}$ gástrico & Alteração da absorção de fármacos que necessitam dissolução com a acidez \\
\hline & $\begin{array}{l}\downarrow \text { Motilidade do trato } \\
\text { digestório } \\
\downarrow \text { Trânsito intestinal } \\
\end{array}$ & $\begin{array}{l}\text { Podem levar a alteração da absorção de medicamentos (p.ex.: Levodopa e } \\
\text { Penicilinas) }\end{array}$ \\
\hline & $\downarrow$ Fluxo sanguíneo esplênico & Absorção de lipossolúveis e fármacos dependentes da $1^{\mathrm{a}}$ passagem hepática \\
\hline \multirow{3}{*}{ Distribuição } & $\downarrow$ Albumina sérica & $\begin{array}{l}\uparrow \text { Fração livre dos fármacos (p.ex.: Fenitoina), } \\
\uparrow \text { Risco de intoxicação }\end{array}$ \\
\hline & $\downarrow$ Massa hídrica e magra & $\begin{array}{l}\downarrow \text { Volume de distribuição dos hidrossolúveis (p.ex.: Digoxina) } \\
\downarrow \text { Dose necessária para atingir concentração plasmática }\end{array}$ \\
\hline & $\uparrow$ Massa de gordura & $\begin{array}{l}\uparrow \text { Volume de distribuição e meia vida de fármacos lipossolúveis (p.ex.: } \\
\text { Benzodiazepínicos) } \\
\uparrow \text { Duração dos efeitos após } 1^{\text {a }} \text { dose e desenvolvimento gradual de toxicidade } \\
\text { com acumulo no tecido adiposo }\end{array}$ \\
\hline \multirow[b]{2}{*}{ Metabolismo } & $\downarrow$ Fluxo sanguíneo hepático & $\begin{array}{l}\downarrow \text { Metabolismo de } 1^{\text {a }} \text { passagem } \\
\downarrow \text { Níveis plasmáticos }\end{array}$ \\
\hline & $\begin{array}{l}\downarrow \text { Massa hepática } \\
\downarrow \text { Atividade do citocromo p450 } \\
\quad \text { (Fase I do metabolismo) }\end{array}$ & $\begin{array}{l}\downarrow \text { Metabolismo oxidativo hepático } \rightarrow \uparrow \text { Meia-vida das drogas metabolizadas } \\
\text { pelo fígado (p.ex.: Quinidina) }\end{array}$ \\
\hline Excreção & $\begin{array}{l}\downarrow \text { Número de glomérulos } \\
\downarrow \text { Massa renal total } \\
\downarrow \text { Fluxo plasmático renal } \\
\downarrow \text { Taxa de Filtração Glomerular } \\
\quad \text { (TFG) } \\
\downarrow \text { Secreção tubular }\end{array}$ & $\begin{array}{l}\downarrow \text { Eliminação dos fármacos de excreção renal } \rightarrow \uparrow \text { meia vida e } \uparrow \text { nível sérico } \\
\text { dos fármacos }\end{array}$ \\
\hline
\end{tabular}

\section{Polifarmácia}

O aumento da expectativa de vida e o crescimento de até $46 \%$ na taxa de envelhecimento populacional durante os últimos dez anos promoveram um aumento na prevalência de Doenças Crônicas Não Transmissíveis (DCNT), predispondo o idoso à polifarmácia, respectivamente ao risco de reações adversas a medicamentos e a prescrição medicamentosa inadequada ${ }^{3,4,6,9,10,11}$.

Atualmente, a polifarmácia é um grande tema a ser discutido na pratica clínica e de extrema relevância à várias especialidades. Existem diversas definições que permeiam desde os aspectos qualitativos até quantitativos. A definição qualitativa é a mais utilizada por estudos norte-americanos, que define polifarmácia como "a prescrição, administração ou uso de mais medicamentos do que está clinicamente indicado". Em contrapartida, os estudos latino-americanos e europeus adotam a definição quantitativa que envolve " $O$ uso simultâneo de vários medicamentos" $" 12$.

Há ainda autores que consideram polifarmácia aqueles em uso de apenas um medicamento se esse não for prescrito $^{12}$.

Com base no critério de prevalência na literatura, considera polifarmácia quando ocorrer a utilização continua de $\geq 5$ tipos de medicamentos ${ }^{4,9,11,12}$.

$\mathrm{Na}$ literatura, há diversas classificações para polifarmácia, subdividindo em polifarmácia menor (uso de 2 a 4 medicamentos) e polifarmácia maior (uso $\geq 5$ medicamentos), em grupos de 1 a 3,4 a 5,6 a 8 e $\geq 9$ medicamentos ou ainda em polifarmácia baixa (uso de 2 a 3 medicamentos), moderada (4 a 5 medicamentos) e alta $(>5 \text { medicamentos })^{12}$.

O uso de vários fármacos pode acarretar em prejuízos a saúde do idoso. Segundo o centro Ibero-americano, para a terceira idade é possível classificar estas interações em 3 níveis: (1) nível menor são os efeitos são usualmente leves, que ocasionam poucas moléstias e podem passar inadvertidamente. Não requerem tratamento adicional; (2) nível moderada podem produzir uma deterioração na situação clinica dos pacientes, requerem associação de tratamento; (3) nível maior: os efeitos são potencialmente ameaçadores para a vida, capazes de produzir um dano permanente ${ }^{9}$.

A utilização de tratamentos não farmacológicos no Brasil é pouco implementada, enquanto que o acesso aos medicamentos é fácil, contribuindo para automedicação e a inúmeras consequências à saúde do idoso 3 . 
As consequências do excesso de medicação são várias como aumento nos custos de saúde tanto para o paciente como para o sistema de saúde, aumento da taxa de eventos adversos da medicação, uso de medicamentos potencialmente inapropriados, não aderência ao tratamento, aumento da incontinência urinária, interação entre drogadroga, interação entre droga-doença, diminuição da capacidade funcional, múltiplas síndromes geriátricas, hospitalização e institucionalização $0^{3,4,12,13}$.

\section{Iatrogenia: reações adversas}

Iatrogenia é qualquer alteração patogênica provocada pela pratica médica e que comumente é representada pela iatrofarmacogenia. A RAM é a principal causa de iatrogenia identificada na prática médica. $\mathrm{O}$ risco de esta acontecer é de até $88 \%$ em idosos que utilizam cinco ou mais medicamentos ${ }^{4,9,12}$.

A reação adversa a medicamentos (RAM) é considerada como uma resposta nociva do organismo e não intencional ao uso de um medicamento cuja dose é normalmente utilizada nos seres humanos. Os idosos são responsáveis por $25 \%$ das admissões hospitalares, seja por reação aguda, subaguda ou crônica devido a RAM. Essas são ainda mais frequentes quando os medicamentos são considerados inapropriados para uso em idosos ${ }^{4,13}$.

Os medicamentos que mais frequentemente causam RAM são Anti-inflamatórios Não Esteroidais (AINEs), betabloqueadores, Inibidores da Enzima Conversora de Angiotensinogenio (IECA), diuréticos, digoxina, antilipidêmicos, depressores do sistema nervoso central, os indutores (fenitoina e carbamazepina) e os inibidores enzimáticos (cimetidina e omeprazol) ${ }^{13}$.

As principais reações adversas em idosos são confusão mental, quedas, hipotensão postural, incontinência urinaria, retenção urinaria e intestinal, sintomas parkinsonianos que mimetizam a doença de Parkinson (p.ex.: tremores, rigidez e lentificação dos movimentos), insônia, entre outros. A morbimortalidade da RAM relacionada ao uso de mais de um medicamento é alta, pois podem ser graves e inclusive fatais em indivíduos mais susceptíveis ${ }^{4,13}$.

\section{Medicamentos potencialmente inapropriados: uma visão mundial sobre as principais listas ou critérios}

As alterações na composição corporal, nas funções renal e hepática provocadas pelo processo do envelhecimento humano geram mudanças farmacocinéticas e farmacodinâmicas em vários medicamentos como já foi discutido anteriormente. Devido a isso, os idosos são considerados os mais susceptíveis aos eventos adversos aos medicamentos (RAM) e ao uso de Medicamentos Potencialmente Inapropriados (MPI).

Os MPI para idosos são fármacos prescritos por profissionais habilitados (médicos), a qual apresenta um risco de causar eventos adversos maiores do que os seus benefícios, mesmo quando há alternativas eficazes. Essas medicações favorecem o surgimento de interações droga-droga e droga-doença, além dos efeitos colaterais indesejáveis que geram impacto sobre a qualidade de vida desses idosos $3,4,6,9,11,14$.

Um fármaco pode ainda ser considerado potencialmente inapropriado pelo risco aumentado de reações adversas, quando o uso do medicamento agrava a sua doença de base ou por falta de evidencias cientificas acerca da eficácia terapêutica, visto que pesquisas clínicas para aprovação na comercialização de fármacos quase nunca tem como população os idosos ${ }^{4,9,11}$.

Visto isso, pesquisadores propuseram instrumentos (listas ou critérios) que possibilitam a detecção de potenciais riscos iatrogênicos causados por medicamentos em idosos. Esses instrumentos, também considerados como um método de prevenção quaternária são de grande utilidade na pratica clínica. Os critérios de Beers foi o primeiro instrumento criado, em 1991, pelo geriatra norte americano Dr. Mark Howard Beers para classificar os principais medicamentos considerados inapropriados para uso em idosos a longo prazo. Beers (1991), descreveu sua lista com um consenso mundialmente conhecido, implementado na prática clínica americana e utilizada em várias pesquisas clinicas de cunho fármaco-epidemiológica ${ }^{3,4,15,16}$.

Em 2012, penúltima versão dos critérios de Beers, houve uma parceria com a American Society of Geriatric que possibilitou atualizações mais frequentes, revisões sistemáticas mais rigorosas e uma maior disseminação desse critério. Além disso, os critérios de Beers passou a dividir os MPI em dois grupos distintos, sendo que em um encontram-se as medicações que devem ser evitadas, independente da doença ou condição clínica do idoso e, em outro grupo as medicações consideradas potencialmente inapropriadas para idosos em determinadas doenças ou síndromes. Atualmente, os Critérios de Beers encontra-se na quinta atualização (versão 1991, 1997, 2003, 2012 e a última em 2015) $)^{17,18}$.

Na revisão de 2015 foi acrescentado uma lista de interações medicamentosas que estão associadas aos danos em idosos, uma relação de medicamentos que devem ser evitados e os medicamentos que devem ter as suas dosagens ajustadas de acordo com a sua função renal. Essa lista pode ser utilizada para qualquer idoso, com exceção daqueles em cuidados paliativos ou que estão em hospices (modalidade de atendimento em Cuidados Paliativos para pacientes em terminalidade $)^{18}$.

O critério STOPP/START, publicado pela primeira vez em 2008 e atualizado em 2014, foi também reconhecido pela European Union Geriatric Medicine Ssociety. Tratase de uma ferramenta baseada em evidência que consegue detectar potenciais omissões na terapia medicamentosa de pacientes idosos. Baseia-se no conjunto de duas listas de MPI, ou seja, critérios STOPP (Screening Tool of Older 
Person's potentially inappropriate Prescriptionst) e START (Screening Tool too Alert doctors to the Right Treatment). Esta lista, desenvolvida para a realidade europeia, é capaz de detectar um número maior de idosos em uso de MPI na Europa Ocidental quando comparado aos critérios de Beers. Isso é justificado pela grande quantidade de medicamentos que são raramente prescritos ou comercializados no continente ${ }^{19,20}$.

A lista PRISCUS é outro instrumento criado para avaliação de MPI. Esse foi criado por pesquisadores alemães em 2010, especificamente para uso na população da Alemanha devido as diferentes drogas que são comercializadas, prescritas e sugeridas nos guias terapêuticos do país. Essa lista foi criada em quatro fases: (1) análise qualitativa de listas de MPI selecionados para idosos de outros países; (2) pesquisa de literatura nas principais bases de dados; (3) desenvolvimento de uma lista preliminar de MPI para idosos, especificamente adaptados ao mercado alemão e (4) geração da lista PRISCUS mediante a consulta de especialistas (método de Delphi modificado). Nesta lista, os medicamentos são categorizados como MPI e aqueles que devem ter a dose ajustada e com monitorização laboratorial ${ }^{21}$.

Recentemente, foi publicado na Geriatric, Gerontologic and Aging, o Consenso Brasileiro de Medicamentos Potencialmente Inapropriados para idosos adaptado a realidade do Brasil. Trata-se do primeiro estudo brasileiro de impacto, voltado para a comercialização e prevalência dos principais medicamentos prescritos na prática clínica do país. O propósito da criação desse instrumento foi melhorar a qualidade e a segurança da prescrição de indivíduos susceptíveis as RAM, ou seja, os idosos ${ }^{22}$.

O Consenso Brasileiro de Medicamentos Potencialmente Inapropriados para idosos tornou-se a solução para carência de critérios adaptados a prática clínica do país, visto que muitos estudos utilizam, principalmente os critérios de Beers para análise de grupos de idosos. Cabe ressaltar que, apenas $60 \%$ dos medicamentos citados nos critérios de Beers são comercializados no Brasil, criando um viés nos resultados de vários estudos brasileiros ${ }^{23}$.

Este estudo realizou a validação de conteúdo dos Critérios de Beers 2012 e STOPP 2006 para obtenção de critérios nacionais de classificação de MPI para idosos. $\mathrm{Na}$ primeira e segunda etapa do estudo, os autores utilizaram o método de Delphi modificada para criar uma lista preliminar de medicamentos (potencialmente inapropriado independente das condições clinicas/doenças e potencialmente inapropriados dependentes de condições clinicas/doenças) e propor sugestões. Posteriormente, um questionário eletrônico foi realizado e cada especialista emitiu sua nota, classificando assim os potencialmente inapropriados quando obtiveram média do Intervalo de Confiança 95\% (IC95\%) $\geq 4,0^{22}$.

As Tabelas 3 e 4 mostram os principais MPI independente das condições clinicas/doenças e dependente das condições clinicas/doenças, respectivamente.

Todas essas listas de MPI são frequentemente utilizadas em grande escala por clínicas geriátricas e centros educacionais, para pesquisa e desenvolvimento de indicadores de qualidade em serviços de saúde.

\section{Efeito placebo-nocebo}

Por definição, efeito placebo-nocebo advém de uma substancia inerte e sem propriedades farmacológicas que está associada a eventos adversos positivos ou negativos. Ambos os efeitos, Placebo e Nocebo, têm origem neurobiológica em relação aos sintomas ou a doença. Sabese que o psicológico de um indivíduo é capaz de modular os processos bioquímicos no sistema nervoso central, influenciando na resposta ao tratamento ${ }^{24}$.

$\mathrm{O}$ efeito placebo corresponde aos eventos adversos positivos de um tratamento empregado. A expectativa positiva de um idoso sobre o tratamento, favorece o surgimento da ansiedade e ao estímulo de vias centrais. Essa resposta psicobiológica ativa a liberação de catecolaminas e outras substâncias bioquímicas que causam um alivio ou melhora dos seus sintomas ${ }^{24,25,26}$.

O efeito Nocebo tem definição oposta, a expectativa negativa e pessimista conduz a liberação de substancias neuroquímicas que medeia a resposta contraria ao placebo, gerando eventos adversos negativos sobre os seus sintomas $^{24,25,26}$.

O efeito placebo-nocebo é atribuído, principalmente a fatores não farmacológicos como relação médicopaciente, desejo de melhora, comportamento e expectativa do paciente no tratamento. Acredita-se que outros fatores estejam envolvidos nesta resposta do efeito placebonocebo, sendo a idade um destes ${ }^{24}$.

Como já discutido anteriormente, o idoso apresenta uma maior vulnerabilidade as reações adversas de um tratamento devido as particularidades do processo do envelhecimento, e isto ocorre com maior frequência em idosos submetidos à terapia antineoplásica. Acredita-se que o uso do placebo em idosos com diagnóstico oncológico possa ser benéfico contra os eventos adversos causados pela quimioterapia. Partindo disso, alguns pesquisadores acreditam que fatores neurobiológicos associados a senescência estejam envolvidos nesta resposta ao placebo. A resposta neurobiológica mediada pelo sistema imune do idoso pode ser um dos principais mecanismos relacionado aos efeitos adversos positivos e negativos e que deve ser mais investigado por estudos clinico randomizados ${ }^{24}$.

Com base em nosso levantamento, alguns estudos mostraram resultados divergentes a essa discussão, evidenciando que o envelhecimento não influencia significativamente sobre o efeito placebo-nocebo, devendo haver cuidado clinico independentemente da idade. No entanto, cabe ressaltar que não há um consenso na literatura sobre a influência da idade no efeito placebo-nocebo. Além disso, existe uma carência de estudos na literatura abordando esta temática no idoso, principalmente sobre o efeito nocebo ${ }^{24,27,28}$. Portanto, para que esse assunto seja mais explorando e aprofundado deve-se realizar novos estudos abordando essa temática no idoso. 
Oliveira HSB, Corradi MLG. Aspectos farmacológicos do idoso: uma revisão integrativa de literatura

Tabela 3 - Medicamentos potencialmente inapropriados independentes das condições clinicas/doenças, segundo o Consenso Brasileiro de Medicamentos Potencialmente Inapropriados para idosos, $2017^{23}$

\begin{tabular}{|c|c|}
\hline Classe & Critério \\
\hline $\begin{array}{l}\text { Sistema Nervoso } \\
\text { Central e } \\
\text { Medicamentos } \\
\text { Psicotrópicos }\end{array}$ & $\begin{array}{l}\text { Antiparkinsonianos: Biperideno, Triexifenidil } \\
\text { Anti-histamínicos de } \mathbf{1}^{\mathrm{a}} \text { geração: Bronfeniramina, Carbinoxamina, Ciproeptadina, Clemastina, Clofeniramina, } \\
\text { Dexclorfeniramina, Difenidramina, Dimenidrinato, Doxilamina, Hidroxizina, Meclizina, Prometazina, Triprolidina } \\
\text { Antipsicóticos de } \mathbf{1}^{\text {a }} \text { geração: Clorpromazina, Flufenazina, Haloperidol, Levomepromazina, Penfluridol, Periciazina, } \\
\text { Pimozida, Pipotiazina, Sulpirida, Tioridazina, Trifluoperazina, Zuclopentixol } \\
\text { Antipsicótico de } \mathbf{2}^{\text {a }} \text { geração: Amissulprida, Aripiprazol, Clozapina, Olanzapina, Paliperidona, Quetiapina, Risperidona, } \\
\text { Ziprasidona } \\
\text { Barbitúricos: Fenobarbital, Tiopental } \\
\text { Benzodiazepínicos: Alprazolam, Bromazepam, Clobazam, Clonazepam, Clordiazepóxido, Cloxazolam, Diazepam, } \\
\text { Estazolam, Flunitrazepam, Flurazepam, Lorazepam, Midazolam, Nitrazepam } \\
\text { Neurolépticos: Hipnóticos } \\
\text { Hipnóticos não benzodiazepínicos: Zolpidem } \\
\text { Antidepressivos tricíclicos terciários: Amitriptilina, Imipramina, Nortriptilina, Clomipramina, Maprotilina } \\
\text { Tioridazina }\end{array}$ \\
\hline $\begin{array}{l}\text { Sistema } \\
\text { Cardiovascular }\end{array}$ & $\begin{array}{l}\text { Bloqueadores alfa-1: Doxazosina, Prazosina, Terazosina } \\
\text { Alfa agonistas de ação central: Clonidina, Metildopa, Reserpina }(>0,1 \mathrm{mg} / \mathrm{dia}) \\
\text { Antiarrítmicos: Amiodarona, Propafenona, Quinidina, Sotalol } \\
\text { Aspirina ( }>150 \mathrm{mg} / \mathrm{dia}) \\
\text { Digoxina }(>0,125 \mathrm{mg} / \mathrm{dia}) \\
\text { Dipiridamol } \\
\text { Diuréticos: Bumetanida, Furosemida, Piretanida } \\
\text { Nifedipino, Espironolactona ( }>25 \mathrm{mg} / \text { dia }) \\
\text { Ticlopidina }\end{array}$ \\
\hline Sistema Endócrino & $\begin{array}{l}\text { Estrógeno: com ou sem progesterona } \\
\text { Andrógenos } \\
\text { Clorpropamida } \\
\text { Antidiabético oral: Glibenclamida } \\
\text { Hormônio do crescimento: Somatropina }\end{array}$ \\
\hline $\begin{array}{l}\text { Sistema } \\
\text { Gastrointestinal }\end{array}$ & $\begin{array}{l}\text { Antiespasmódicos gastrointestinais: Hiosciamina, Escopolamina } \\
\text { Loperamida, Codeína } \\
\text { Metoclopramida } \\
\text { Óleo mineral (via oral) } \\
\text { Inibidores de bomba de prótons: Omeprazol, Pantoprazol, Lanzoprazol, Rabeprazol, Esomeprazol, Tenatoprazol por } \\
>8 \text { semanas }\end{array}$ \\
\hline $\begin{array}{l}\text { Sistema } \\
\text { Musculoesquelético }\end{array}$ & $\begin{array}{l}\text { Relaxantes musculares: Carisoprodol, Ciclobenzaprina, Orfenadrina } \\
\text { AINEs: Aspirina ( }>325 \mathrm{mg} / \text { dia), Diclofenaco, Etodolaco, Fenoprofeno, Ibuprofeno, Cetoprofeno, Meloxicam, Nnaproxeno, } \\
\text { Piroxicam } \\
\text { Indometacina } \\
\text { Cetorolaco } \\
\text { Corticosteroides sistêmicos (>3 meses): Betametasona, Budesonida, Deflazacorte, Dexametasona, Fludrocortisona, } \\
\text { Hidrocortisona, Metilprednisolona, Prednisolona, Prednisona } \\
\text { Colchicina: (uso prolongado) } \\
\text { Opioides: Alfentanila, Fentanila, Hidromorfona, Metadona, Morfina, Nalbufina, Oxicodona, Petidina, Remifentanila, } \\
\text { Sufentanila (uso prolongado) } \\
\text { Petidina: Dolantina, Meperidina }\end{array}$ \\
\hline Diversos & $\begin{array}{l}\text { Nitrofurantoína } \\
\text { Teofilina }\end{array}$ \\
\hline
\end{tabular}


Tabela 4 - Medicamentos potencialmente inapropriados dependentes das condições clinicas/doenças, segundo o Consenso Brasileiro de Medicamentos Potencialmente Inapropriados para idosos, $2017^{23}$

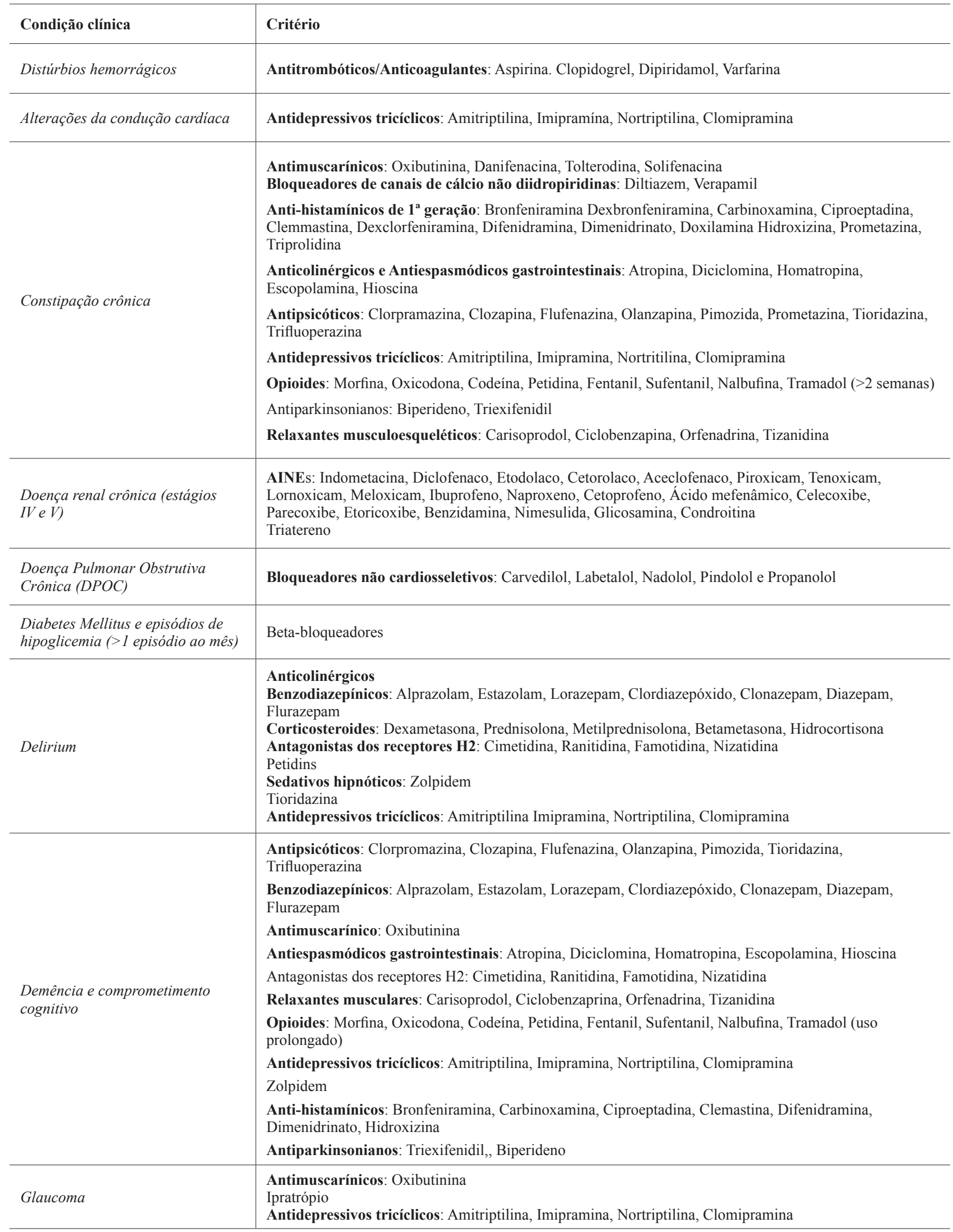


Oliveira HSB, Corradi MLG. Aspectos farmacológicos do idoso: uma revisão integrativa de literatura

Tabela 4 - Medicamentos potencialmente inapropriados dependentes das condições clinicas/doenças, segundo o Consenso Brasileiro de Medicamentos Potencialmente Inapropriados para idosos, $2017^{23}$

continuação

\begin{tabular}{|c|c|}
\hline Condição clínica & Critério \\
\hline Gota & Diuréticos tiazídicos: Hidroclorotiazida, Clortalidona \\
\hline Insuficiência cardíaca & $\begin{array}{l}\text { Bloqueadores de canais de cálcio não diidropiridinas: Diltiazem, Verapamil } \\
\text { Cilostazol } \\
\text { AINEs: Indometacina, Diclofenaco, Etodolaco, Cetorolaco, Aceclofenaco, Piroxicam, Tenoxicam, } \\
\text { Lornoxicam, Meloxicam, Ibuprofeno, Naproxeno, Cetoprofeno, Ácido mefenâmico, Celecoxibe, } \\
\text { Parecoxibe, Etoricoxibe, Benzidamina, Nimesulida, Glicosamina, Condroitina } \\
\text { Tiazolidinedionas: Pioglitazona }\end{array}$ \\
\hline $\begin{array}{l}\text { História de câncer de mama ou } \\
\text { tromboembolismo venoso }\end{array}$ & Estrógenos e Análogos: Etinilestradiol, Estradiol, Estriol, Promestrieno, Dietilestilbestrol, Tibolona \\
\hline História de queda ou fraturas & $\begin{array}{l}\text { Anticonvulsivantes: Fenobarbital, Primidona, Fenitoína, Clonazepam, Carbamazepina, Oxcarbazepina, } \\
\text { Ácido valpróico, Vigabatrina, Lamotrigina, Topiramato, Gabapentina, Pregabalina } \\
\text { Anti-histamínicos de } \mathbf{1}^{\text {a }} \text { geração: Brongeniramina, Dexbronfeniramina, Carbinoxamina, Ciproeptadina, } \\
\text { Clemastina, Dexclorfeniramina, Difenidramina, Hidroxizina, Prometazina, Triprolidina } \\
\text { Antipsicóticos: Clorpramazina, Flufenazina, Haloperidol, Pimozida, Tioridazina, Aripiprazol, Clozapina, } \\
\text { Olanzapina, Paliperidona, Quetiapina, Risperidona, Ziprasidona } \\
\text { Benzodiazepínicos } \\
\text { Hipnóticos não benzodiazepínicos: Zolpidem } \\
\text { Opioides: Morfina, Oxicodona, Codeína, Petidina, Fentanil, Sufentanil, Nalbufina, Tramadol (uso } \\
\text { prolongado) } \\
\text { Inibidores seletivos de receptação de serotonina: Fluoxetina, Citalopram, Paroxetina, Sertralina, } \\
\text { Fluvoxamina, Escitalopram, Venlafaxina } \\
\text { Antidepressivos tricíclicos: Amriptilina, Imipramina, Nortriptilina, Clomipramina }\end{array}$ \\
\hline História de ulcera péptica & $\begin{array}{l}\text { AINEs não seletivos da COX-2: Indometacina, Diclofenaco, Etodolaco, Cetorolaco, Aceclofenaco, } \\
\text { Piroxicam, Tenoxicam, Lornoxicam, Meloxicam, Ibuprofeno, Naproxeno, Cetoprofeno, Ácido } \\
\text { mefenâmico, Celecoxibe, Parecoxibe, Etoricoxibe, Benzidamina, Nimesulida, Glicosamina, Condroitina } \\
\text { AINEs seletivos da COX-2: Celecoxibe, Parecoxibe, Etoricoxibe }\end{array}$ \\
\hline Hipertensão (HAS) & $\begin{array}{l}\text { AINEs não seletivos da COX-2 em idosos com HAS moderada à grave: Indometacina, Diclofenaco, } \\
\text { Etodolaco, Cetorolaco, Aceclofenaco, Piroxicam, Tenoxicam, Lornoxicam, Meloxicam, Ibuprofeno, } \\
\text { Naproxeno, Cetoprofeno, Ácido mefenâmico, Celecoxibe, Parecoxibe, Etoricoxibe, Benzidamina, } \\
\text { Nimesulida, Glicosamina, Condroitina }\end{array}$ \\
\hline Insônia & $\begin{array}{l}\text { Descongestionantes orais: Pseudoefedrina, Fenilefrina } \\
\text { Estimulantes: Metilfenidato } \\
\text { Teobrominas: Teofilina, Cafeína }\end{array}$ \\
\hline $\begin{array}{l}\text { Sintomas que afetam o trato } \\
\text { urinário }\end{array}$ & Anticolinérgicos orais e inalatórios (em homens) \\
\hline Doença de Parkinson & $\begin{array}{l}\text { Antipsicóticos de } 1^{\text {a }} \text { geração: Clorpromazina, Flufenazina, Haloperidol, Piimozida, Tioridazina } \\
\text { Antipsicóticos de } 2^{\text {a }} \text { geração: Aripiprazol, Clozapina, Olanzapina, Paliperidona, Quetiapina, Risperidona, } \\
\text { Ziprasidona } \\
\text { Metoclopramida } \\
\text { Prometazina }\end{array}$ \\
\hline Hipertensão postural persistente & Vasodilatadores: Hidralazina, Minoxidil \\
\hline Convulsões & $\begin{array}{l}\text { Antipsicóticos atípicos: Clozapina, Olanzapina } \\
\text { Antipsicóticos convencionais: Clorpromazina, Tioridazina } \\
\text { Bupropiona } \\
\text { Maprotilina } \\
\text { Tramadol }\end{array}$ \\
\hline Hiponatremia $(<130 \mathrm{Meq} / \mathrm{L})$ & $\begin{array}{l}\text { Inibidores seletivos da recaptação de serotonina: Fluoxetina, Citalopram, Paroxetina, Sertralina, } \\
\text { Fluvoxamina, Escitalopram }\end{array}$ \\
\hline História de síncope & $\begin{array}{l}\text { Anticolinesterásicos: Donepezila, Rivastigmina, Galantamina } \\
\text { Alfa-bloqueadores periférico: Doxazosina, Prazosina, Terazosina } \\
\text { Clorpromazina } \\
\text { Olanzapina } \\
\text { Tioridazina } \\
\text { Antidepressivos tricíclicos terciários: Amitriptilina, Clmipramina, Imipramina }\end{array}$ \\
\hline
\end{tabular}




\section{CONCLUSÃO}

Conclui-se que, o processo do envelhecimento fisiológico tem grande impacto sobre a farmacologia do idoso, evidenciando a relevância do tema abordado. Vários países implementaram em sua prática clínica instrumentos para a prevenção de reações adversas à medicamentos e para cuidado com o uso de medicamentos potencialmente inapropriados. Atualmente, o estudo da farmacologia do idoso associado a utilização desses instrumentos são de grande impacto em serviços e auxiliam os profissionais área da saúde para que possam atuar de forma interdisciplinar com qualidade na saúde do idoso.

CONFLITO DE INTERESSE: Não há qualquer conflito de interesse dos autores em relação a este manuscrito.

CONTRIBUIÇÕES DOS AUTORES: Concepção ou delineamento do estudo: Oliveira HSB; Aquisição, análise e interpretação dos dados: Oliveira HSB, Corradi MLG; Elaboração do estudo: Oliveira HSB; Revisão do estudo: Corradi MLG; Aprovação final da versão ao ser publicada: Oliveira HSB, Corradi $M L G$.

\section{REFERÊNCIAS}

1. Moraes EN. Atenção à saúde do idoso: aspectos conceituais. Brasília: Organização Pan-Americana da Saúde; 2012. p.98. Disponível em: http://apsredes.org/pdf/Saude-doIdoso-WEB1.pdf .

2. Manso MEG. E a vida, como vai? Avaliação da qualidade de vida de um grupo de idosos portadores de doenças crônicas não transmissíveis vinculados a um programa de promoção da saúde [dissertação]. São Paulo: Pontifícia Universidade Católica, Programa de Estudos Pós-graduados em Gerontologia; 2009. Disponível em: http://livros01. livrosgratis.com.br/cp118972.pdf.

3. Donis ACG, Oliveira HSB, Sousa JRP. Prescrição medicamentosa potencialmente inapropriada (PMPI): aplicação dos critérios de Beers 2015 em um grupo de idosos do setor suplementar de saúde [monografia]. São Paulo: Centro Universitário São Camilo; 2017.

4. Vera ECBA. Terapia medicamentosa do idoso: fatores de influência [dissertação]. São Paulo: Pontifícia Universidade Católica, Programa de Estudos Pós-graduados em Gerontologia; 2017. Disponível em: https://sapientia. pucsp.br/bitstream/handle/19829/2/Elaine\%20Cristina\%20 Biffi\%20Alonso\%20Vera.pdf.

5. Nobrega OT, Karnikowski MGO. A terapia medicamentosa no idoso: cuidados na medicação. Cien Saude Coletiva. 2005;10(2):303-13. doi: http://dx.doi.org/10.1590/S141381232005000200008 .

6. Manso MEG, Biffi ECA, editoras. Manual da LEPE Liga de Estudos do Processo do Envelhecimento. São Paulo: Martinari; 2014.

7. IMS Institute for Healthcare Informatics. The global use of medicines: outlook through 2017. Parsippany, NJ; 2017. Available from: http://www.quotidianosanita.it/allegati/ allegato1501906.pdf.

8. Silva WJM, Ferrari, CKB. Metabolismo mitocondrial, radicais livres e envelhecimento. Rev Bras Geriatr Gerontol. 2011;14(3):441-51. doi: http://dx.doi.org/10.1590/S180998232011000300005.

9. Silva R, Schmidt OF, Silva S. Polifarmácia em geriatria. Rev AMRIGS. 2012;56(2):164-74. Disponível em: http:// www.amrigs.org.br/revista/56-02/revis.pdf.

10. Oliveira HSB, Sousa JRP, Donis ACG, Umeda E, Manso MEG. Risco eminente de quedas por prescrição potencialmente inadequada (PPI) em um grupo de idosos vinculados a um plano de saúde na cidade de São Paulo, Brasil. In: $10^{\circ}$ Congresso Paulista de Geriatria e
Gerontologia; abr. 2017. Anais do GERP - Gerontologia. p.493.

11. Manso MEG, Biffi ECA, Gerardi TJ. Prescrição inadequada de medicamentos a idosos portadores de doenças crônicas em um plano de saúde no município de São Paulo, Brasil. Rev Bras Geriatr Gerontol. 2015;18(1):151-64. doi: http:// dx.doi.org/10.1590/1809-9823.2015.14056.

12. Carvalho MFC. A polifarmácia em idosos no município de São Paulo - Estudo SABE - Saúde, Bem-estar e Envelhecimento [dissertação]. São Paulo: Faculdade de Saúde Pública da Universidade de São Paulo, Programa de Pós-graduação em Saúde Pública; 2007. Disponível em: http://www.fsp.usp.br/sabe/Teses/Maristela.pdf.

13. Secoli SR. Polifarmácia: interações e reações adversas no uso de medicamentos por idosos. Rev Bras Enferm. 2010;63(1):136-40. doi: http://dx.doi.org/10.1590/S003471672010000100023 .

14. Sousa-Munoz RL, Ibiapina GR, Gadelha CS, Maroja JLS. Prescrições geriátricas inapropriados e polifarmacoterapia em enfermarias de clínica médica de um hospital-escola. Rev Bras Geriatr Gerontol. 2012;15(2):315-24. doi: http:// dx.doi.org/10.1590/S1809-98232012000200014.

15. Quinalha JV, Correr CJ. Instrumentos para avaliação da farmacoterapia do idoso: uma revisão. Rev Bras Geriatr Gerontol. 2010;13(3):487-99. doi: http://dx.doi. org/10.1590/S1809-98232010000300014.

16. Beers MH, Ouslander JG, Rollingher I, Reuben DB, Brooks J, Beck JC. Explicit criteria for determining inappropriate medication use in nursing home residents. Arch Intern Med. 1991;151(9):1825-32. doi: http://dx.doi.org/10.1001/ archinte.1991.00400090107019.

17. American Geriatrics Society updated Beers Criteria for potentially inappropriate medication use in older adults. J Am Geriatr Soc. 2012;60(4):616-31. http://doi.org/10.1111/ j.1532-5415.2012.03923.x.

18. American Geriatrics Society 2015 updated Beers Criteria for potentially inappropriate medication use in older adults. J Am Geriatr Soc. 2015;63(11):2227-46. doi: http://doi. org/10.1111/jgs.13702.

19. Delgado-Silveira E, Muñoz-García M, Montero-Errasquin B, Sánchez-Castellano C, Gallagher PF, Cruz-Jentoft AJ. Prescripción inapropriada de medicamentos em los pacientes mayores: los critérios STOPP/START. Rev Esp Geriatr Gerontol. 2009;44:273-9. doi: http://doi. org/10.1016/j.regg.2009.03.017.

20. O'Mahony D, O'Sullivan D, Byrrne S, O'Connor MN, Ryan C, Gallagher P. STOPP/START criteria for potentially 
inappropriate prescribing in older people: version 2. Age Ageing. 2014;44(2):213-8. doi: http://doi.org/10.1093/ ageing/afu145.

21. Holt S, Schmiedl S, Thurmann PA. Potentially inappropriate medications in the elderly: the PRISCUS list. Deutsch Arztebl Int. 2010;107(31-32):543-51. doi: http://doi. org/10.3238/arztebl.2010.0543.

22. Oliveira MG, Amorim WW, Oliveira CRB, Coqueiro HL, Gusmão LC, Passos LC. Consenso brasileiro de medicamentos potencialmente inapropriados para idosos. Geriatr Gerontol Aging. 2017;10(4):168-81. doi: http://doi. org/10.5327/Z2447-2115201616000054.

23. Lopes LM, Figueiredo TP, Costa SC, Reis AMM. Utilização de medicamentos potencialmente inapropriados por idosos em domicilio. Cien Saúde Coletiva. 2016;21(11):3429-38. doi: http://doi.org/10.1590/1413-812320152111.14302015.

24. Foster JC, Le-Rademacher JG, Feliciano JL, Gajra A, Seisler DK, DeMatteo R et al. Comparative "nocebo effects" in older patients enrolled in cancer therapeutic trials: observations from a 446-patient cohort. Cancer. 2017;123(21):4193-8. doi: http://doi.org/10.1002/cncr.30867.

25. Enck P, Benedetti F, Schedlowski M. New insights into the placebo and nocebo responses. Neuron. 2008;59(31):195206. doi: http://doi.org/10.1016/j.neuron.2008.06.030.

26. Klaric M, Mandic V, Lovric S, Coric MK, Zovko N. Placebo and nocebo effects and their significance in clinical practice. Med Glas (Zenica). 2017;14(1):16-24. doi: http:// doi.org/10.17392/892-16.

27. Cruz M, Hui D, Parsons HA, Bruera E. Placebo and nocebo effects in randomized double blind clinical trials of agentes for the treatment of fatigue in advanced cancer patients. Cancer. 2010;116(3):766-74. doi: http://doi.org/10.1002/ cncr.24751.

28. Hunter AM, Leuchter AF, Morgan ML, Cook IA, Abrams M, Siegman B, et al. Neurophysiologic correlates of side effects in normal subjects randomized to venlafaxine or placebo. Neuropsychopharmacology. 2005;30(4):792-9. doi: http://doi.org/10.1038/sj.npp.1300652.

Recebido em: 14.11 .17

Aceito em: 16.01 .18 